\title{
Pharmaciana
}

Vol. 8, No.1, May 2018, Page. 135-144

ISSN: 2088 4559; e-ISSN: 24770256

DOI: $10.12928 /$ pharmaciana.v8i1.9308

\section{The formulation of carvedilol transdermal patch with resin gum as rate control}

\author{
Kori Yati", Septiana Tri Pamungkas \\ Fakultas Farmasi dan Sains, Universitas Muhammadiyah Prof. DR. HAMKA \\ Jl. Delima II/IV Perumnas Klender Jakarta Timur
}

Submitted: 19-03-2018

Reviewed: 27-03-2018

Accepted: 25-04-2018

\begin{abstract}
Carvedilol is widely prescribed for long-term hypertension treatment. It is rapidly absorbable by oral administration, but its bioavailability is merely about $20 \%$ in humans. Drug delivery is therefore imperative to overcome this weakness. One form of transdermal drug delivery system is a patch. Transdermal patch is composed of various systems, for instance, a reservoir that uses a rate control layer to manage the rate of drug release. This research aimed to observe the effect of using resin gum as the control of drug release rate on the physical characteristics and release of carvedilol in a transdermal patch. The patches were prepared in 5 formulas with different quantities of resin gum, namely 50, 100, 150, 200, and $250 \mathrm{mg}$. Afterward, they were evaluated physically, and their dissolution and diffusion rates were analyzed. The results showed that resin gum with concentrations of $150 \mathrm{mg}$ and $200 \mathrm{mg}$ was physically qualified for rate control. Besides, the results of dissolution and diffusion tests revealed that transdermal patches with $150 \mathrm{mg}$ of resin gum exhibited the best drug release and penetration.
\end{abstract}

Keywords: carvedilol, rate control, reservoir, resin gum, transdermal patch

Corresponding author:

Kori Yati

Fakultas Farmasi dan Sains, Universitas Muhammadiyah Prof. DR. HAMKA

Jl. Delima II/IV Perumnas Klender Jakarta Timur

Email: koriyati@uhamka.ac.id 


\section{INTRODUCTION}

Carvedilol is a non-selective $\beta$-blocker that can block $\alpha-1, \beta-1$, and $\beta-2$ adrenergic receptors. It is widely prescribed for long-term hypertension treatment. Clinically, it has been proven not only to treat hypertension but also to be used in myocardial infarction and congestive heart failure. It is rapidly absorbable when administered orally. However, its bioavailability in humans is merely around $20 \%$ because it undergoes the first-pass metabolism that converts it into its inactive form and, thereby, provides no therapeutic effect. Due to the low oral bioavailability, it requires a repeated administration that may reduce medication compliance (Ahad et al., 2015).

A drug delivery system is required to overcome these weaknesses. Carvedilol has a low molecular weight $(406.5 \mathrm{Da})$ and a favorable $\log \mathrm{P}(4.115)$ with $\mathrm{t}_{1 / 2}=6$ hours, allowing it to penetrate the lipophilic layer (Vora et al., 2013). Transdermal drug delivery system (TDDS) is a delivery system that maintains the effectiveness of drug release over an extended period, reducing the intensity of administration and side effects associated with oral administration routes (Patel et al., 2012). One form of TDDS is a patch. Transdermal patches are pharmaceutical preparations with a variety of size containing one or more active ingredients intended to be applied to the skin and accommodate these ingredients to enter the systemic circulation after passing through the skin barrier (Premjee et al., 2011). TDDS has several advantages, including convenience in drug delivery, pain reduction, and easy treatment in case of systemic toxicity (Alexander et al., 2012).

As a dosage form, transdermal patch consists of many systems, i.e., adhesive system (using adhesive polymers), matrix system (using polymers as a matrix/drug container), reservoir system (using polymers as drug containers and controls of drug release rate,) and micro-reservoir system. This study prepared transdermal patches with reservoir system due to its several advantages, for instance, reservoirs (drug carriers) have separate adhesive layer and a control layer of drug release rate (Alexander et al., 2012; Saroha et al., 2011). Moreover, if the carvedilol patches were prepared with a matrix system, then a combination of hydrophobic and hydrophilic polymers would be needed and, at the same time, complicate the time of preparation. Transdermal patch with reservoir system is composed of active substance, matrix polymer, polymer as rate control, penetration enhancer, plasticizer (preventing the formation of a brittle film layer), release liner (protecting the adhesive layer during storage), and backing layer (the outermost layer that protects the patch) (Premjeet et al., 2011).

Polymers as rate control have an essential role in a transdermal patch with reservoir systems. An example of these polymers is resin gum. It is mainly used as an emulsifier and a stabilizer in the production of colors, paints, inks, and aromatic emulsions in food and cosmetic industry, primarily because it is one of the relatively cheap and readily available polymers (Coppen, 1995). Resin gum is a hydrophobic polymer that can form a good film layer and release the drug gradually, which justifies its application as excellent rate control. Previous studies prove that it can create a functional film layer with the addition of $40 \%$ of dibutyl phthalate (hydrophobic) as a plasticizer. The drug diffusion through this film provides zero-order release over an extended period at a controlled level (Mundada and Avari, 2009). This reason has encouraged researchers to observe the effect of using resin gum as rate control on the physical characteristics and the release of carvedilol in a transdermal patch.

\section{MATERIALS AND METHODS}

\section{Materials}

The materials used in this research were carvedilol (Sigma) and resin gum (Biuma Bhakti).

Formulation of transdermal patches (Mundada and Avari, 2009; Rajhpur and Lakshmi, 2015) The five formulas used to prepare the carvedilol transdermal patches are presented in Table I. 
Table I. Formulas of carvedilol transdermal patch

\begin{tabular}{ccccc}
\hline Formulas & $\begin{array}{c}\text { Carvedilol } \\
(\mathbf{m g})\end{array}$ & $\begin{array}{c}\text { 3\% HPMC } \\
\text { K100M Gel } \\
(\mathbf{m g})\end{array}$ & $\begin{array}{c}\text { Resin Gum } \\
(\mathbf{m g})\end{array}$ & $\begin{array}{c}\text { Dibutyl phthalate } \\
(\%, \text { from the amount of } \\
\text { resin gum })\end{array}$ \\
\hline F1 & 13 & 100 & 50 & 40 \\
F2 & 13 & 100 & 100 & 40 \\
F3 & 13 & 100 & 150 & 40 \\
F4 & 13 & 100 & 200 & 40 \\
F5 & 13 & 100 & 250 & 40 \\
\hline
\end{tabular}

\section{Preparation of carvedilol-containing gel}

HPMC K100M was dispersed in distilled water. The dispersion solution was stirred until homogeneous using a magnetic stirrer and then left to rest for \pm 24 hours at room temperature until it expanded and gelatinized. The carvedilol was dissolved in methanol, added to the gel, and stirred until homogeneous. Afterward, the gel in which carvedilol was present was observed organoleptically and evaluated for content uniformity.

\section{Preparation of rate control layer}

The resin gum was dissolved in chloroform until it reached a concentration of $10 \% \mathrm{w} / \mathrm{v}$. It was then added with $40 \%$ dibutyl phthalate, stirred until homogeneous, and placed in a Petri dish. After a film layer was formed, it was evaluated organoleptically and tested experimentally for thickness, weight uniformity, folding endurance, and water vapor transmission rate.

\section{Preparation of transdermal patches}

The gel containing carvedilol was placed on the backing layer, and the resin gum layer was put on top of it. Afterward, the transdermal patch was closed with a release liner and tested for in vitro drug release and diffusion.

\section{Evaluations}

Organoleptic analysis of carvedilol-containing gel (Sonjoy et al., 2011; Rajhpur and Lakshmi, 2015)

This evaluation included visual observation on the shape, color, and odor of the gel.

Content uniformity analysis of carvedilol-containing gel (Sonjoy et al., 2011; Rajhpur and Lakshmi, 2015)

This test was performed by pouring the gel into a volumetric flask. The gel was added with 10 $\mathrm{mL}$ of methanol and stirred in a shaker bath for 24 hours. Then, it was added with phosphate buffer solution (pH 7.4) up to $100 \mathrm{~mL}$. The absorbent was measured with UV-Vis spectrophotometer at a wavelength of $286 \mathrm{~nm}$.

Organoleptic analysis of rate control layer (Sonjoy et al., 2011)

This evaluation included visual observation on the shape, color, and odor of the rate control layer.

Thickness measurement of rate control layer (Sonjoy et al., 2011)

The thickness of rate control layer was measured using a caliper. The result represented the average thickness of the rate control layers of three different patches. 
Weight uniformity analysis of rate control layer (Sonjoy et al., 2011)

Three rate control layers of each formula were weighed one by one with an analytical scale. The variation of their weight was calculated.

Folding endurance test of rate control layer (Sonjoy et al., 2011)

This test was performed by folding the rate control layer repeatedly for 300 times until it was broken or folded. The number of folds that could be applied to the layer without breaking it was selected as the degree of folding endurance.

Water vapor transmission rate (WVTR) analysis of rate control layer (Sonjoy et al., 2011)

This test used glass vials as a transmission cell. The vials were washed and then dried in the oven. Afterward, 1 gram of calcium chloride was placed in them, and the rate control was set on the edge with the help of an adhesive. The accurate weight of the vials was recorded. They were put in a desiccator containing a saturated potassium chloride solution with a relative humidity of $63 \%$. The vials were removed after 72 hours. The water vapor transmission rate was calculated using Eq. 1 .

$W V T R=\frac{W \times L}{S}$

where:

$\mathrm{W}=$ Weight difference or reduction (gram)

$\mathrm{L}=$ Thickness of transdermal patch $(\mathrm{cm})$

$\mathrm{S}=$ Area $\left(\mathrm{cm}^{2}\right)$

In vitro drug release test of transdermal patch (Sonjoy et al., 2011)

The drug release was measured using a U.S.P dissolution tester at a temperature of $37^{\circ} \mathrm{C} \pm 1^{\circ} \mathrm{C}$ and stirred at $50 \mathrm{rpm}$. The transdermal patches were placed on the glass object with the help of cyanoacrylate adhesive substance so that the drug could not separate from the glass object. The glass object was inserted into a chamber containing $900 \mathrm{~mL}$ of phosphate buffer solution ( $\mathrm{pH}$ 7.4). Afterward, $5 \mathrm{ml}$ of the solution in the chamber was discharged every hour up to the twentieth hour and diluted with phosphate buffer solution $(\mathrm{pH}$ 7.4). The absorbent was read using a UV-Vis spectrophotometer at a wavelength of $286 \mathrm{~nm}$.

\section{In vitro drug penetration test of transdermal patch (Sonjoy et al., 2011)}

This test used the Frans diffusion cell tool. The transdermal patches were placed on the Millipore membrane (pore size $=0.22 \mu \mathrm{m}$ ) in the Franz diffusion cell tool. Millipore membranes were inserted between the donor and receptor compartments. The receptor compartment contained $100 \mathrm{~mL}$ of phosphate buffer solution ( $\mathrm{pH}$ 7.4). This buffer was stirred with a magnetic stirrer at $100 \mathrm{rpm}$ and a temperature of $37^{\circ} \mathrm{C} \pm 1^{\circ} \mathrm{C}$. It was drawn by $5 \mathrm{~mL}$ per predefined time interval for 24 hours. The solution was diluted with phosphate buffer solution $(\mathrm{pH}$ 7.4) and then analyzed using a UV-Vis spectrophotometer at a wavelength of $286 \mathrm{~nm}$.

\section{Statistical analysis}

The physical characteristics of the rate control layer and the transdermal patch, as identified from the evaluations, were analyzed using One-way ANOVA with t $\alpha=0.05$. 


\section{RESULTS AND DISCUSSION}

The organoleptic properties and content uniformity of the carvedilol-containing gel

The gel base used in this research was a high viscosity grade of hydroxypropyl methylcellulose (HPMC) with a concentration of 3\%. The organoleptic test results of the HPMC gel are presented in Table II.

Table II. The organoleptic properties of HPMC gel

\begin{tabular}{cc}
\hline Organoleptic Characteristics & Results \\
\hline Shape & Gel \\
Color & Transparent (colorless) \\
Odor & Odorless \\
\hline
\end{tabular}

The carvedilol content in each part of the gel has to be uniform. Therefore, the content uniformity of HPMC gel in which carvedilol is present is necessary to evaluate. The carvedilolcontaining HPMC gel was sampled by $100 \mathrm{mg}$, dissolved in phosphate buffer solution (pH 7.4), and measured using a UV-Vis spectrophotometer at a wavelength of $286 \mathrm{~nm}$. As presented in Table III, the results indicate that the carvedilol content in the tested HPMC gel meets the standard of content uniformity, i.e., no deviation above 15\% (Kemenkes RI, 2014).

Table III. The results of content uniformity test of carvedilol-containing HPMC gel

\begin{tabular}{cc}
\hline No & Results (\%) \\
\hline 1. & 100.5 \\
2. & 99.8 \\
3. & 99.5 \\
4. & 100.3 \\
5. & 100.2 \\
6. & 99.7 \\
7. & 99.8 \\
8. & 100.2 \\
9. & 100.4 \\
10. & 99.6 \\
Mean & 100.0 \\
\hline
\end{tabular}

\section{The evaluation results of the rate control layer}

The rate control layer was prepared with resin gum using the film casting solvent method, which evaporated the solvent used in the preparation of the rate control layer. The film layer formed in this process was evaluated organoleptically. As summarized in Table IV, the results showed that formulas 3 and 4 had the most favorable organoleptic properties because they produced non-friable and nonsticky rate control layers. These characteristics are influenced by the amount of resin gum used in each formula. The higher the amount of the resin gum used to a certain extent, the better the film layer (Mundada and Avari, 2009).

The next evaluation was the thickness measurement of the film layer using a micrometer scrub. The number of polymers used in the preparation of the rate control layer affects the thickness of the film layer (Mundada and Avari, 2009; Babu et al., 2012). The results, as presented in Table V, affirmed that the amount of resin gum used in the preparation could produce film layers with significantly different thickness $(\mathrm{p}<0.05)$.

Aside from thickness, the research analysis included the weight uniformity evaluation of the rate control layer. Three transdermal patches of each formula were weighed using an analytical scale. The 
amount of resin gum used as the former of film layer affects the weight of the dosage form (Mundada and Avari, 2009). As listed in Table VI, the results proved that the amount of resin gum used in the preparation could produce rate control layer with significantly different weights $(p<0.05)$.

Table IV. The results of the organoleptic test of the resin gum-based rate control layer

\begin{tabular}{|c|c|}
\hline Formulas & Results \\
\hline Formula 1 & $\begin{array}{c}\text { Shape: Friable thin film layer } \\
\text { Color: Transparent brown } \\
\text { Odor: Odorless }\end{array}$ \\
\hline Formula 2 & $\begin{array}{c}\text { Shape: Slightly friable, thin film layer } \\
\text { Color: Transparent brown } \\
\text { Odor: Odorless }\end{array}$ \\
\hline Formula 3 & $\begin{array}{c}\text { Shape: Thin film layer } \\
\text { Color: Transparent brown } \\
\text { Odor: Odorless }\end{array}$ \\
\hline Formula 4 & $\begin{array}{l}\text { Shape: Thin film layer } \\
\text { Color: Transparent brown }\end{array}$ \\
\hline Formula 5 & $\begin{array}{c}\text { Odor: Odorless } \\
\text { Shape: Slightly sticky, thin film layer } \\
\text { Color: Transparent brown } \\
\text { Odor: Odorless }\end{array}$ \\
\hline
\end{tabular}

Table V. The thickness of the resin gum-based rate control layer

\begin{tabular}{cc}
\hline Formulas & Results $(\boldsymbol{\mu m})$ \\
\hline Formula 1 & $83.5 \pm 0.45$ \\
Formula 2 & $95.6 \pm 1.01$ \\
Formula 3 & $130.2 \pm 0.58$ \\
Formula 4 & $167.8 \pm 0.34$ \\
Formula 5 & $201.7 \pm 0.48$ \\
\hline
\end{tabular}

Table VI. The weight uniformity of the resin gum-based rate control layer

\begin{tabular}{cc}
\hline Formulas & Results $(\mathbf{m g})$ \\
\hline Formula 1 & $53.86 \pm 0.28$ \\
Formula 2 & $102.55 \pm 0.30$ \\
Formula 3 & $153.75 \pm 0.31$ \\
Formula 4 & $203.30 \pm 0.55$ \\
Formula 5 & $252.48 \pm 0.32$ \\
\hline
\end{tabular}

The folding endurance test was performed by folding the patches 300 times in the same section. A good film can withstand at least 300 folds (Barhate et al., 2011). The evaluation results showed that the film layer of formula 1 was only able to survive 238 folds, while the one created using formula 2 survived 273 folds. Similar case applied to formula 5 where the film layer endured 280 folds. However, the film layers of formulas 3 and 4 were able to withstand the three-hundredth fold without any damage. Such endurance to folding was caused by the use of $40 \%$ of dibutyl phthalate as a plasticizer (Mundada and Avari, 2009). The amount of the resin gum used in the formula potentially affects the endurance of the film layer to folding. The higher the amount of the polymers used as the 
former of the film layer, the stronger its endurance to folding (Barhate et al., 2011). The number of folding endured by the film layers continually increased from formula 1 to formulas 3 and 4 that used $150 \mathrm{mg}$ and $200 \mathrm{mg}$ of resin gum. However, this number decreased when the formula used $250 \mathrm{mg}$ of resin gum because it resulted in a slightly sticky layer. These evaluation results suggested that formulas 3 and 4 produced the best film layers of resin gum. Furthermore, they affirm that the amount of resin gum in a formula can create significantly different endurance to folding $(\mathrm{p}<0.05)$.

The water vapor transmission rate (WVTR) test was performed by measuring the amount of water vapor that passed through the film layer. This amount is influenced by the thickness of said film layer (Premjeet et al., 2011). As summarized in Table VII, the test results showed that a thicker film layer allowed a smaller amount of water to pass through. They also proved that the amount of resin gum used in the preparation of the rate control layer produced different thickness and, therefore, significantly different water vapor transmission rate $(\mathrm{p}<0.05)$.

Table VII. The water vapor transmission rate of the resin gum-based rate control layer

\begin{tabular}{cc}
\hline Formulas & Results $\left(\mathbf{g . c m} / \mathbf{c m}^{\mathbf{2}}\right)(\mathbf{7 2}$ hours $)$ \\
\hline Formula 1 & $7.73 \pm 0.07$ \\
Formula 2 & $7.11 \pm 0.12$ \\
Formula 3 & $6.38 \pm 0.08$ \\
Formula 4 & $5.85 \pm 0.04$ \\
Formula 5 & $5.02 \pm 0.10$ \\
\hline
\end{tabular}

\section{The evaluation results of the carvedilol transdermal patch}

The dissolution test was performed to identify the ability of the transdermal patch to release active substance from its dosage form. It tested the carvedilol transdermal patches prepared with formulas 3 and 4 because they were the best and most eligible according to the physical evaluation results of the resin gum-based film layer. The results of the dissolution test of the carvedilol transdermal patch are summarized in Table VIII. The drug release in a transdermal dosage form with reservoir system is influenced by the properties of the polymer, such as rate control, the thickness of the rate control layer, and the characteristics of the active ingredient (Premjeet et al., 2011). Based on the dissolution test results, formula 3 released more active substance compared to formula 4 despite the less significant thickness difference between their rate control layers. This finding affirms that a thicker rate control layer decreases the speed of the active substance to penetrate; in other words, the active ingredient takes a longer time to exit the dosage form (Sonjoy et al., 2011). The statistical analysis results indicated that the two formulas yielded inconsiderably different percentage of dissolved active ingredient ( $\mathrm{p}>0.05)$.

In addition to the dissolution test, the evaluation of carvedilol patch also included diffusion test. It was performed using Franz diffusion cell. The Millipore membrane used to simulate skin layer in this test had to be impregnated first with isopropyl myristate to produce oily membrane and mimic the structure of stratum corneum. The drug diffusion test started with identifying the optimum impregnation time in 10, 30, 45, 60, and 75 minutes. As presented in Table IX, the percentages showed that the optimum impregnation time was 10 minutes (Hendradi and Martodihardjo, 1997).

The diffusion test was performed to identify the ability of active substances to penetrate the stratum corneum on the skin to be able to enter the systemic circulation. It tested the carvedilol transdermal patches prepared with formulas 3 and 4 due to their most favorable organoleptic properties and degrees of folding endurance. It used a diffusion tool that was connected to a peristaltic pump. The receptor compartment containing $330 \mathrm{~mL}$ of phosphate buffer solution $(\mathrm{pH}$ 7.4) was conditioned at a temperature $37^{\circ} \mathrm{C} \pm 1^{\circ} \mathrm{C}$ by running water with the same temperature into the water jacket. The previous study used Eudagrit L to form the rate control layer and Span 80 as a penetration enhancer, and it resulted in 90\% dissolved active substance in 24 hours (Tanwar et al., 2007). 
Table VIII. The dissolution test results of the carvedilol transdermal patch

\begin{tabular}{ccc}
\hline Time (hour) & $\begin{array}{c}\text { Results (\%) } \\
\text { F3 }\end{array}$ & $\begin{array}{c}\text { Results (\%) } \\
\text { F4 }\end{array}$ \\
\hline 0 & $0 \pm 0$ & $0 \pm 0$ \\
1 & $8.98 \pm 0.13$ & $5.25 \pm 0.19$ \\
2 & $15.67 \pm 0.05$ & $7.15 \pm 0.07$ \\
3 & $22.37 \pm 0.14$ & $15.67 \pm 0.14$ \\
4 & $33.90 \pm 0.23$ & $23.54 \pm 0.12$ \\
5 & $40.86 \pm 0.21$ & $30.78 \pm 0.24$ \\
6 & $52.35 \pm 0.18$ & $40.44 \pm 0.13$ \\
8 & $60.75 \pm 0.13$ & $48.92 \pm 0.21$ \\
10 & $68.34 \pm 0.16$ & $52.45 \pm 0.07$ \\
14 & $73.54 \pm 0.18$ & $60.34 \pm 0.03$ \\
24 & $80.21 \pm 0.16$ & $68.62 \pm 0.13$ \\
\hline
\end{tabular}

Table IX. The optimization of millipore membrane

\begin{tabular}{cc}
\hline $\begin{array}{c}\text { Time } \\
\text { (minute) }\end{array}$ & $\begin{array}{c}\text { \% average impregnation } \\
(\boldsymbol{\%})\end{array}$ \\
\hline 10 & $9.08 \pm 0.3872$ \\
30 & $14.89 \pm 0.4979$ \\
45 & $22.10 \pm 0.7349$ \\
60 & $28.68 \pm 0.2573$ \\
75 & $31.13 \pm 0.7556$ \\
\hline
\end{tabular}

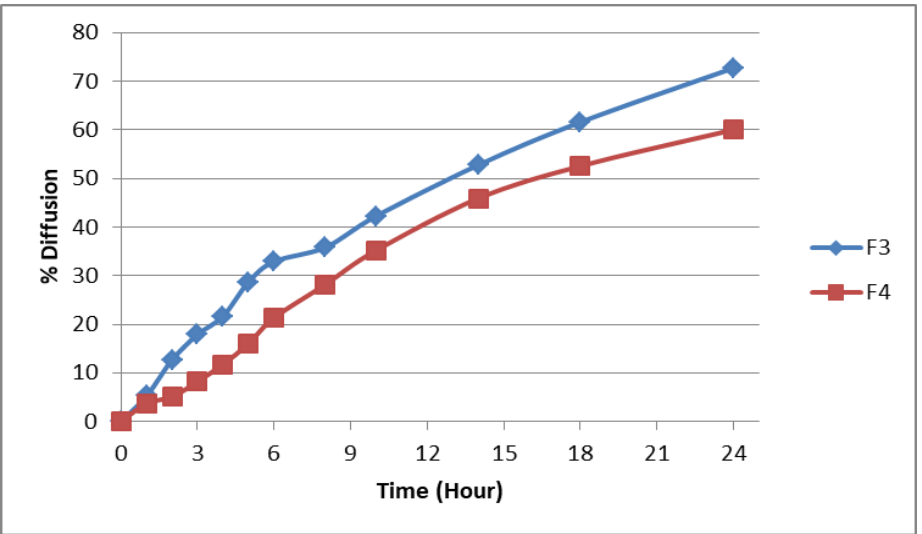

Figure 1. The diffusion test results of carvedilol transdermal patches prepared with formula 3 and formula 4

The percentage of the diffused active substance by the carvedilol transdermal patch prepared with formula 3 was higher than formula 4, as illustrated in Figure I. This difference was caused by the thicker rate control layer in formula $4(72.7 \%)$ compared to formula $3(60.01 \%)$, which prevented drug from penetrating to the rate control layer and reduced the amount of the diffused drug. A long drug release is also influenced by the hydrophobicity of the rate control layer. Resin gum is a hydrophobic polymer; therefore, medicinal materials can hardly penetrate it and diffuse out of a dosage system. In 
this case, only a smaller amount of drug can penetrate the skin and enter the systemic circulation (Babu et al., 2012). Compared to the previous study (Tanwar et al., 2007), the diffusion test results showed that transdermal patches with resin gum as the rate control layer exhibited a reasonably good diffusion even without the addition of penetration enhancer. The statistical analysis results showed that carvedilol patches prepared with formulas 3 and 4 produced insignificantly different percentage of diffused active substance $(\mathrm{p}>0.05)$.

\section{CONCLUSIONS}

The results indicate that a higher concentration of resin gum as a rate control affects the physical properties of the formed rate control layer. The amount of resin gum used in the preparation also determines the results of the dissolution and diffusion test of the carvedilol transdermal patch. Formula 3 , with $150 \mathrm{mg}$ of resin gum, is the best formula.

\section{REFERENCES}

Ahad, A., Al-Jenoobi, F., Al-Mohizea, A.M., Akhtar, N., Raish, M., and Aqil, M., 2015. Systemic delivery of $\beta$-blockers via transdermal route for hypertension, Saudi Pharmaceutical Journal, 23(6): 587-602.

Alexander, A., Dwivedi, S., Ajazuddin, Giri T.K., Saraf, S., and Tripathi D.K., 2012. Approaches for Breaking the Barriers of Drug Permeation Through Transdermal Drug Delivery, Journal of Controlled Release, 164(1): 26-40.

Babu, G.D., Sagar, K.C., and Bhootr M.R., 2012. Design and evaluation of valsartan transdermal patches. International Journal of Research in Ayurveda and Pharmacy, 3(3), 462-464.

Barhate, S.D., and Potdar, M.B., 2011. Formulation of Transdermal Patch of Carvedilol Using Novel Polymers, Pelagia Research Library, 2(2): 185-189.

Coppen, J.J.W., 1995. Non-Wood Forest Products 6: Gums, Resins and Latexes of Plant Origin. Food and Agriculture Organization of The United Nations, Rome, 65.

Hendradi, E., and Martodihardjo, S., 1997. Difusi astemizol melewati membran isopropil miristat, Majalah Farmasi Indonesia, 8(4): 143-149.

Kementrian Kesehatan Republik Indonesia (Kemenkes RI), 2014. Farmakope Indonesia Edisi V. Jakarta: Direktorat Jenderal Pengawasan Obat dan Makanan, pp.1526-1529.

Mundada, A.S., and Avari, J.G., 2009. Permeability studies of damar batu free films for transdermal application, Chemical Industry and Chemical Engineering Quarterly, 15(2), 83-88.

Patel, H., Bhimani B., and Patel G., 2012. Transdermal Drug Delivery System As Prominent Dosage Forms for the Highly Lipophilic Drugs, International Journal of Pharmaceutical Bio-Science, 1(3): 42-65.

Premjeet, S., Bilandi, A., Sahil, K., and Akanksha M., 2011. Transdermal Drug Delivery System (Patches), Applications In Present Scenario, International Journal Of Research In Pharmacy And Chemistry, 1(4): 1139-1151.

Rajhpur, A., and Lakshmi, P.K., 2015. Development of reservoir type transdermal drug delivery system of alfuzosin hydrochloride for the treatment of benign prostate hyperplasia. Der Pharmacia Lettre, 7(4): 31-39.

Saroha, K., Yadav, B., and Sharma, B., 2011. Transdermal Patch: A Discrete Dosage Form. International, Journal of Current Pharmaceutical Research, 3(3): 98-108.

Sonjoy M., Thimmasetty, R., and Kilarimath, 2011. Formulation and Evaluation of Carvedilol Transdermal Patches, International Research Journal of Pharmacy, 2(1), 237-248.

Tanwar, Y.S., Cauchan, C.S., and Sharma, A., 2007. Development and Evaluation of Carvedilol Transdermal Patches, Acta Pharma, 57: 151-159.

Vora, N., Lin, S., and Madan, P.L., 2013. Development and in-vitro evaluation of an optimized carvedilol transdermal therapeutic system using experimental design approach. Asian Journal of Pharmaceutical Sciences, 8, 28-38. 
